PROCEEDINGS OF THE

AMERICAN MATHEMATICAL SOCIETY

Volume 136, Number 10, October 2008, Pages 3503-3511

S 0002-9939(08)09535-X

Article electronically published on June 10, 2008

\title{
ABSOLUTELY BOUNDED MATRICES AND UNCONDITIONAL CONVERGENCE
}

\author{
MILAN HLADNIK \\ (Communicated by N. Tomczak-Jaegermann)
}

\begin{abstract}
We characterize the so-called absolutely bounded matrices in terms of the (strong) unconditional convergence of their standard decompositions. There is a similar characterization of absolutely compact matrices, and both characterizations are closely related to some natural multiplication operators. Rudiments of the duality theory for the algebra of all absolutely bounded matrices are included.
\end{abstract}

\section{INTRODUCTION}

We shall consider infinite matrices as complex functions on $\mathbb{N} \times \mathbb{N}$. The commutative algebra of all bounded complex functions on $\mathbb{N} \times \mathbb{N}$, i.e. the algebra of all matrices with uniformly bounded entries equipped with Schur or the entrywise product, will be denoted by $B_{\infty}$. Although this product is usually denoted by $x * y$ or $x \circ y$, we shall simply write $x y$, as is used for the product of functions and, when necessary, use the notation $x \circ y$ for the ordinary matricial product. Other operations on matrices will also be taken entrywise, e.g. $|x|=\left(\left|x_{i j}\right|\right)$ if $x=\left(x_{i j}\right)$. Of course, $\|x\|_{\infty}=\sup \left\{\left|x_{i j}\right| ; i, j \geq 1\right\}$ is the natural norm on $B_{\infty}$.

We are interested in subalgebras of $B_{\infty}$ connected with operators. Let us denote by $B$ the set of all infinite complex matrices $x=\left(x_{i j}\right)$ which represent bounded linear operators on $l^{2}$ via the usual matrix multiplication. We shall write $\|x\|$ for the operator norm of $x \in B$. Those elements in $B$ which give compact operators on $l^{2}$ will be denoted by $K$ and its Banach dual, the trace class, by $T$.

By a component of an infinite matrix $x$ we mean the matrix with only one nonzero entry taken from $x$, i.e. $x_{(i, j)}=x_{i j} e_{(i, j)}$ for $i, j \in \mathbb{N}$ where $e_{(i, j)}$ is the usual matrix unit. Choose a square enumeration of the matrix units; that is, arrange them in the usual order: $e_{(1,1)}, e_{(2,1)}, e_{(2,2)}, e_{(1,2)}, e_{(3,1)}, e_{(3,2)}, \ldots$. Always using this order we can write matrix units with a single index: $e_{(k)}, k=1,2, \ldots$. Then the appropriate components of an arbitrary matrix $x$ are denoted simply by $x_{(k)}, k=1,2, \ldots$. The series $x=\sum_{k} x_{(k)}$ will be called the standard decomposition of $x$.

Received by the editors June 13, 2007.

2000 Mathematics Subject Classification. Primary 47B49; Secondary 47L20.

Key words and phrases. Absolutely bounded matrices, standard decomposition, unconditional convergence, absolutely compact matrices, duality.

This work was supported in part by the Ministry of Higher Education, Science and Technology of Slovenia.

(C)2008 American Mathematical Society Reverts to public domain 28 years from publication 
In the following three lemmas we rewrite known facts (folklore results) about infinite matrices in the new notation and give no proof, which can be found elsewhere.

Lemma 1.1. Let $x$ be an infinite matrix with components $x_{(k)}, k=1,2, \ldots$. Then the following assertions are equivalent:

(i) $x \in B$;

(ii) $\sup _{n \geq 1}\left\|\sum_{k=1}^{n} x_{(k)}\right\|<\infty$;

(iii) the series $x=\sum_{k} x_{(k)}$ converges in the strong operator topology in $B$.

Moreover, if $x \in B$, then $\|x\|=\sup _{n}\left\|\sum_{k=1}^{n^{2}} x_{(k)}\right\|$.

Note that for $x \in K$ the series $x=\sum_{k} x_{(k)}$ is really a Schauder decomposition since it can easily be seen that in this case the series is norm convergent.

Lemma 1.2. An infinite matrix $x$ belongs to $K$ if and only if the standard decomposition $x=\sum_{k} x_{(k)}$ converges in the operator norm $\|\cdot\|$.

An analoguous result holds for the trace class operators.

Lemma 1.3. An infinite matrix $y$ belongs to $T$ if and only if the standard decomposition $y=\sum_{k} y_{(k)}$ converges in the trace norm $\|\cdot\|_{T}$.

Our main idea is to explore in a similar way the unconditional convergence of the standard decomposition in various operator topologies.

An infinite complex matrix $\left(x_{i j}\right)$ such that $\left(\left|x_{i j}\right|\right)$ defines a bounded linear operator on $l^{2}$ is usually said to be absolutely bounded (see, e.g., [5]). Besides giving a general description of absolutely bounded matrices and introducing a related concept of absolutely compact matrices, in Section 2 we characterize both kinds of matrices in terms of Schur multiplication by matrices with uniformly bounded elements (see Theorems 2.5 and 2.7). Then we show in Section 3 that a matrix $x$ is absolutely compact (respectively, absolutely bounded) if and only if the standard decomposition is norm (respectively, strongly) unconditionally convergent (see Theorems 3.1 and 3.2). This answers a question of Sunder [5] about the characterization of absolutely bounded matrices. We relate operator properties of infinite matrices to some of the well known notions from the theory of unconditional summability. Following the same analogy we give some natural characterizations of absolutely bounded and absolutely compact matrices in terms of various multiplication operators (Theorem 3.3). All these observations are quite elementary, but they shed, perhaps, a new light onto the otherwise classical material about infinite matrices. In Section 4 we briefly consider a little less familiar unconditional convergence of the standard decomposition in the trace norm (which is dual to the operator norm) and in a weaker norm (which is dual to the absolutely bounded norm).

\section{Absolute Boundedness}

Let us call a matrix $x=\left(x_{i j}\right)$ absolutely bounded if $|x|=\left(\left|x_{i j}\right|\right) \in B$, and let us denote by $B_{a}$ the set of all absolutely bounded matrices. We shall write $|x| \leq|y|$ if $\left|x_{i j}\right| \leq\left|y_{i j}\right|$ for every $i, j \in \mathbb{N}$. Also, define $\|x\|_{a}=\||x|\|$ for $x \in B_{a}$ and note that $\|x\|_{a}=\|x\|$ if $x \geq 0$ (i.e. if $x_{i j} \geq 0$ for every $i, j \in \mathbb{N}$ ).

Proposition 2.1. Let $b \in B_{\infty}, y, z \in B_{a}$ and $|x| \leq|y|$. Then we have:

(a) $x \in B_{a}$ and $\|x\| \leq\|x\|_{a} \leq\|y\|_{a}$;

(b) $y+z \in B_{a}$ and $\|y+z\|_{a} \leq\|y\|_{a}+\|z\|_{a}$; 
(c) $y z \in B_{a}$ and $\|y z\|_{a} \leq\|y\|_{a}\|z\|_{a}$;

(d) by $\in B_{a}$ and $\|b y\|_{a} \leq\|b\|_{\infty}\|y\|_{a}$.

Proof. First of all, since $|y| \in B$ and $|x| \leq|y|$, then (by definition of the operator norm of a matrix $x$ acting in the usual way on a vector $\zeta \in l^{2}$ ) we have

$$
\|x \zeta\|_{2} \leq\left\|\left|x\|\zeta \mid\|_{2} \text { and }\||x| \zeta\|_{2} \leq\left\|\left|x\left\|\zeta\left|\left\|_{2} \leq\right\|\right| y\right\| \zeta\right|\right\|_{2}\right.\right.
$$

where $|\zeta|=\left(\left|\zeta_{k}\right|\right)$ if $\zeta=\left(\zeta_{k}\right) \in l^{2}$. Hence, (a) follows from (1) and (b) follows directly from (a) as $|y+z| \leq|y|+|z|$ holds for every $y, z$. The submultiplicativity of the norm in (c) is a well known inequality for Schur products (see, e.g., Satz III in [4]) and easily obtained directly. Finally, (d) can be immediately derived from the definition of the operator norm.

By Proposition 2.1(a) every absolutely bounded matrix belongs to $B$, i.e. $B_{a} \subset$ $B$, and by (b) and (c), $B_{a}$ is a subalgebra in $B$. Moreover, by (d) $B_{a}$ is an ideal in $B_{\infty}$ with respect to Schur multiplication. Hence, $B_{a}$ is also a Schur ideal in $B$ (as $\|x y\|_{a} \leq\|x\|_{\infty}\|y\|_{a} \leq\|x\|\|y\|_{a}$ for $x \in B, y \in B_{a}$ ). By (a), (b), and (c) the function $\|\cdot\|_{a}$ defines an algebra norm, usually called the absolute bounded norm, on $B_{a}$, so that $B_{a}$ is a normed algebra. It is useful to relate this norm to the standard decomposition.

Proposition 2.2. Let $x=\sum_{k} x_{(k)}$ and $y=\sum_{k} y_{(k)}$ be the standard decompositions for $x, y \in B_{a}$. Then the following is true:

(a) $|x|=\sum_{k}\left|x_{(k)}\right|$ is the standard decomposition for $|x|$;

(b) $\left\|\sum_{k=1}^{n} x_{(k)}\right\|_{a} \leq\|x\|_{a}$ for every $n \in \mathbb{N}$;

(c) $\left\|x-\sum_{k=1}^{n} x_{(k)}\right\|_{a}=\left\||x|-\sum_{k=1}^{n}\left|x_{(k)}\right|\right\|$ for every $n \in \mathbb{N}$;

(d) $\left\|x-\sum_{k=1}^{n} x_{(k)}\right\|_{a} \leq\left\|y-\sum_{k=1}^{n} y_{(k)}\right\|_{a}$ for every $n \in \mathbb{N}$ if $|x| \leq|y|$.

Proof. All assertions follow easily from definitions or Proposition 2.1. Note, for example, that $\left|x_{(k)}\right|=|x|_{(k)}$ in (a), $\left|\sum_{k=1}^{n} x_{(k)}\right|=\sum_{k=1}^{n}|x|_{(k)} \leq|x|$ in (b), and $\left|x-\sum_{k=1}^{n} x_{(k)}\right|=\left|\sum_{k=n+1}^{\infty} x_{(k)}\right|=\sum_{k=n+1}^{\infty}|x|_{(k)}=|x|-\sum_{k=1}^{n}|x|_{(k)}$ in (c) and (d).

Note that it follows from (c) that $x=\sum_{k} x_{(k)}$ is a Schauder decomposition of $x$ in $B_{a}$ if and only if $|x|=\sum_{k}|x|_{(k)}$ is a Schauder decomposition of $|x|$ in $B$ (or in $\left.B_{a}\right)$.

The next result is proved in [1] in a more general situation. So we shall give no direct proof here.

Theorem 2.3. $B_{a}$ is a Banach algebra in the norm $\|\cdot\|_{a}$.

Proof. One can produce the proof along the standard lines; however, see also 1 , Theorem 3.2, the case $r=1$.

Remark 2.4. Note that $B_{a}$ is not closed in the usual operator norm in $B$, as we see from the example $u=\bigoplus_{n=1}^{\infty} u_{n} / \sqrt[4]{n}$ where $u_{n}=\frac{1}{\sqrt{n}}\left(\omega^{j k}\right)_{j, k=1}^{n}, \omega$ being a primitive $n$-th root of the unity (see Example 2.8 in [3]). Then $u_{n}$ is a unitary matrix while $\left|u_{n}\right|$ is a positive matrix with all entries equal to $1 / \sqrt{n}$, and so $\left\|\left|u_{n}\right|\right\|=\sqrt{n}$ for every $n$. It follows that $\left\|u-\bigoplus_{n=1}^{m} u_{n} / \sqrt[4]{n}\right\| \rightarrow 0$ as $m \rightarrow \infty$ and, hence, $u \in K \subset B$ while $|u|=\bigoplus_{n=1}^{\infty}\left|u_{n}\right| / \sqrt[4]{n} \notin B$, since $\left\|\left|u_{n}\right|\right\| / \sqrt[4]{n}=\sqrt[4]{n} \rightarrow \infty$ as $n \rightarrow \infty$ and, consequently, $u \notin B_{a}$. 
Our first characterization of the elements in $B_{a}$ is given in terms of the Schur product for various kinds of infinite matrices. By $\chi_{S}$ we denote the matrix which is the characteristic function of a subset $S \subset \mathbb{N} \times \mathbb{N}$.

Theorem 2.5. Let $x$ be an infinite matrix. The following assertions are equivalent:

(i) $x \in B_{a}$;

(ii) $b x \in B$ for every $b \in B_{\infty}$;

(iii) $x \chi_{S} \in B$ for every subset $S \subset \mathbb{N} \times \mathbb{N}$;

(iv) $c x \in B$ for every $c=\left(c_{i j}\right)$ with $c_{i j} \in\{-1,1\}$ for each $(i, j)$.

Proof. The implication (i) $\Longrightarrow$ (ii) follows from Proposition 2.1(d), (ii) $\Longrightarrow$ (iii) is trivial and (iii) $\Longrightarrow$ (iv) is clear, since for every $c=\left(c_{i j}\right) \in B_{\infty}$ with $c_{i j} \in\{-1,1\}$ for each $(i, j)$, we have $x(c+e) \in B$ where $e$ is the matrix with each entry equal to 1 (note that $x e=x \in B$ ). Finally, if (iv) holds, then not only $c x \in B$ but also $c y \in B$ and $c z \in B$ for every \pm 1 matrix $c$, where $y$ and $z$ are the real and the imaginary parts of $x$. So, $|y|,|z| \in B$ and, since $|x| \leq|y|+|z|$, (i) follows by Proposition 2.1(a).

Note that in the case $x \in B_{a}$ we have in fact $b x \in B_{a}$ for every $b \in B_{\infty}$ and that both assertions are equivalent.

In the same way as we introduced absolutely bounded matrices we can also define $K_{a}=\{x ;|x| \in K\}$. Elements in $K_{a}$ will be called absolutely compact matrices. Obviously, $K_{a} \subset B_{a}$. Let $F$ be the set of all matrices of finite rank and $F_{0}$ the set of all matrices with finitely many nonzero components. We shall denote the closure of a subset $M \subset B_{a}$ in $B$ or $B_{a}$ by $\bar{M}$ and $\bar{M}^{a}$, respectively.

The following proposition is almost entirely proven in [3] in slightly different notation (see Theorem 2.6 and Example 2.8).

Proposition 2.6. Let $F_{0}, F$ and $K_{a}$ be as above. Then we have:

(a) $K_{a}$ is a closed Schur ideal in $B_{a}$;

(b) $F_{0} \subset F \subset K_{a} \subset K \cap B_{a} \subset K$ with all inclusions proper;

(c) $\overline{F_{0}}=\bar{F}=K$ and ${\overline{F_{0}}}^{a}=\bar{F}^{a}=K_{a}$.

Proof. Only (a) needs a justification. If $x \in B_{a}$ and $y \in K_{a}$, then $x y \in B_{a}$. By Proposition 2.2(c) we have $\left\||x y|-\sum_{k=1}^{n}\left|x_{(k)} y_{(k)}\right|\right\|=\left\|x y-\sum_{k=1}^{n} x_{(k)} y_{(k)}\right\|_{a}$ $=\left\|x\left(y-\sum_{k=1}^{n} y_{(k)}\right)\right\|_{a} \leq\|x\|_{a}\left\|y-\sum_{k=1}^{n} y_{(k)}\right\|_{a}=\|x\|_{a}\left\||y|-\sum_{k=1}^{n}\left|y_{(k)}\right|\right\|$. Since the right-hand side converges to 0 by Lemma 1.2, we see, by Lemma 1.2 again, that $|x y| \in K$ or $x y \in K_{a}$, and the set $K_{a}$ is a Schur ideal in $B_{a}$. It is closed in $B_{a}$ as we see by using the inequality $\||x|-|y|\| \leq\|x-y\|_{a}$ for $x, y \in B_{a}$.

Note that $K \cap B_{a}$ is also a Schur ideal in $B_{a}$ and that it is closed in $B_{a}$. It would be nice to have an independent description of its elements. We now give a characterization of elements in $K_{a}$.

Theorem 2.7. Let $x$ be an infinite matrix. The following assertions are equivalent:

(i) $x \in K_{a}$;

(ii) $b x \in K$ for every $b \in B_{\infty}$;

(iii) $x \chi_{S} \in K$ for every subset $S \subset \mathbb{N} \times \mathbb{N}$;

(iv) $c x \in K$ for every $c=\left(c_{i j}\right)$ with $c_{i j} \in\{-1,1\}$ for each $(i, j)$.

Proof. If $x \in K_{a}$, we have $\left\|x-\sum_{k=1}^{n} x_{(k)}\right\|_{a}=\left\||x|-\sum_{k=1}^{n}|x|_{(k)}\right\| \rightarrow 0$ for $n \rightarrow \infty$ by Lemma 1.2 and Proposition 2.2(c) and also $\left\|b\left(x-\sum_{k=1}^{n} x_{(k)}\right)\right\| \rightarrow 0$ for every 
$b \in B_{\infty}$ by Proposition 2.1. But $b \sum_{k=1}^{n} x_{(k)}=\sum_{k=1}^{n}(b x)_{(k)} \in F_{0}$ and, hence, $b x \in \overline{F_{0}}=K$. Thus, (i) implies (ii). Other implications follow in the same way as in the proof of Theorem 2.5.

Again, $x \in K_{a}$ if and only if $b x \in K_{a}$ for every $b \in B_{\infty}$. Also, since $b x \in K$ for every $b \in B_{\infty}$ only if $x \in K_{a}$, and since $K_{a} \neq K$ by Proposition 2.6(b), we see that $K$ is not a Schur ideal in $B_{\infty}$.

\section{UnCONDITIONAL CONVERGENCE}

Recall that the unconditional convergence of a series $x=\sum_{k} x_{k}$ in a Banach space means that for every permutation $\sigma: \mathbb{N} \rightarrow \mathbb{N}$ the series $x=\sum_{k} x_{\sigma(k)}$ converges in the norm of the given space. There are several other characterizations of this notion including subseries convergence, sign convergence, bounded multiplier convergence, etc., and also some of the appropriate weak notions, such as weak subseries convergence or weak sign convergence, are equivalent to the unconditional convergence in norm (the proof is nontrivial; see [2], Chapter 1).

The following simple theorem gives a characterization of the elements of $K_{a}$ in terms of norm convergence of the standard decomposition.

Theorem 3.1. For a matrix $x$ with the standard decomposition $x=\sum_{k} x_{(k)}$ the following assertions are equivalent:

(i) $x \in K_{a}$;

(ii) $x=\sum_{k} x_{(k)}$ is unconditionally convergent in the operator norm;

(iii) $|x|=\sum_{k}|x|_{(k)}$ is convergent in the operator norm.

Proof. If $x \in K_{a}$, then $b x \in K$ for every $b \in B_{\infty}$ by Theorem 2.7. Hence, the series $b x=\sum_{k} b_{(k)} x_{(k)}$ converges in $K$ for every $b \in B_{\infty}$. This is equivalent to the unconditional convergence of the series $x=\sum_{k} x_{(k)}$ in $K$ by Theorem 1.6 in [2]. Further, (iii) follows immediately from (ii) since there is $b \in B_{\infty}$ such that $|x|=b x$. Finally, the convergence in (iii) obviously implies $|x| \in K$ by Lemma 1.2.

With Theorem 3.1 in mind, we can easily explain Theorem 2.7 in terms of the unconditional convergence: (ii) corresponds to bounded multiplier convergence of the standard decomposition in norm, (iii) means norm subseries convergence, and (iv) is related to sign convergence (see Theorem 1.9 in [2]).

Replacing norm convergence with convergence in the strong operator topology one obtains an analoguous characterization for $B_{a}$ (instead of $K_{a}$ ). Moreover, this is still true if one replaces the strong operator topology with the weak, or even the ultraweak, topology on $B$.

Before stating the theorem note that in [3] a matrix $x=\left(x_{i j}\right)$ is called absolutely summable if $\sum_{i, j}\left|x_{i j}\right|<\infty$ and that the set of all absolutely summable matrices is denoted by $A S$. It follows that for $x \in A S$ the standard decomposition is absolutely convergent in the operator norm, and hence, for example, $A S \subset K_{a}$. The space $A S$ is a Banach algebra in the norm $\|x\|_{A S}=\sum_{i, j}\left|x_{i j}\right|$; in fact, $A S=l^{1}(\mathbb{N} \times \mathbb{N})$. Note also that $A S$ is a predual of $B_{\infty}$ and the dual of $B_{0}=\left\{x=\left(x_{i j}\right) ; \lim _{n \rightarrow \infty} \| x-\right.$ $\left.\sum_{i, j=1}^{n} x_{(i, j)} \|_{\infty}=0\right\}$. For later use we define the summing functional $t$ on $A S$ by $t(z)=\sum_{i, j} z_{i j}, z \in A S$.

Theorem 3.2. For a matrix $x$ with the standard decomposition $x=\sum_{k} x_{(k)}$ the following assertions are equivalent: 
(i) $x \in B_{a}$;

(ii) $x=\sum_{k} x_{(k)}$ is unconditionally convergent in the strong (resp. weak, ultraweak) operator topology;

(iii) $|x|=\sum_{k}|x|_{(k)}$ is convergent in the strong (resp. weak, ultraweak) operator topology;

(iv) $\sum_{i, j}\left|\eta_{i} x_{i j} \xi_{j}\right|<\infty$ for every $\xi=\left(\xi_{i}\right), \eta=\left(\eta_{j}\right) \in l^{2}$;

(v) $\sum_{i, j}\left|x_{i j} y_{i j}\right|<\infty$ for every $y=\left(y_{i j}\right) \in T$.

Proof. If $x \in B_{a}$, then $b x \in B$ for every $b \in B_{\infty}$ by Theorem 2.5. Hence, for each $\zeta \in l^{2}$ the series $b x \zeta=\sum_{k} b_{(k)} x_{(k)} \zeta$ converges in $l^{2}$ for every $b \in B_{\infty}$. This is equivalent to the unconditional convergence of the series $x \zeta=\sum_{k} x_{(k)} \zeta$ in $l^{2}$ by Theorem 1.6 in [2. Further, (iii) follows immediately from (ii) since there is $b \in B_{\infty}$ such that $|x|=b x$, and the convergence in (iii) implies $|x| \in B$ by Lemma 1.1. Hence, (i) and the "strong" parts of (ii) and (iii) are equivalent. Moreover, (iv) can be interpreted as the weak unconditional convergence of the series $\sum_{k} x_{(k)} \xi$ for every $\xi \in l^{2}$, i.e. the unconditional convergence of the scalar series $\sum_{k}\left\langle x_{(k)} \xi, \eta\right\rangle$ for every pair $\xi, \eta \in l^{2}$, which is of course the same as the absolute convergence of this series. Thus, (iv) is a consequence of (ii). On the other hand, (iv) is the same as $\sum_{i, j}\left\langle|x|_{(i, j)}|\xi|,|\eta|\right\rangle=\sum_{k}\left\langle|x|_{(k)}|\xi|,|\eta|\right\rangle<\infty$ for every $\xi, \eta \in l^{2}$. Since this means that we have the (unconditional) weak operator convergence in (iii), it follows that $|x| \in B$, i.e. (i) holds. Finally, note that if $x \in B_{a}, y \in T$ and if we take $b \in B_{\infty}$ such that $b y=|y|$, then we have $b|x| \in B$ and $\sum_{i, j}\left|x_{i j} y_{i j}\right|=\sum_{i, j} b_{i j}|x|_{i j} y_{i j}=\operatorname{trace}\left(b|x| \circ y^{T}\right)<\infty$ where $\circ$ means the usual matricial product. Hence, (v) follows from (i). Conversely, suppose that (v) holds. This is the same as $\sum_{i, j}\left|t\left(x_{(i, j)} y\right)\right|<\infty$ or $\sum_{k}\left|t\left(x_{(k)} y\right)\right|<\infty$. Note that the duality between $B$ and $T$ is given by the bilinear functional trace $\left(x \circ y^{T}\right)$ for $x \in$ $B, y \in T$. Since trace $\left(x \circ y^{T}\right)=t(x y)$, the condition $\sum_{k}\left|t\left(x_{(k)} y\right)\right|<\infty$ is equivalent to the unconditional convergence of the standard decomposition $x=\sum_{k} x_{(k)}$ in the weak-* topology, i.e. the ultraweak operator topology, on $B$. A similar conclusion holds for $|x|$ instead of $x$. Therefore, also the "ultraweak" parts of (ii) and (iii) are equivalent to (i).

Note that (v) means that $x y \in A S$ for every $y \in T$, while (iv) means that $x y \in A S$ for every rank-one matrix $y=\left(\eta_{i} \xi_{j}\right) \in B$ with $\left(\xi_{i}\right),\left(\eta_{j}\right) \in l^{2}$.

Having the above characterization we see that for $x \in K_{a}$ (or $x \in B_{a}$ ) the exact order of taking components does not matter, and we can simply write $x=\sum_{i, j} x_{(i, j)}$ with the convergence in the norm (or the strong) operator sense.

For appropriate matrices $x$ define the multiplication operators $u_{x}: T \rightarrow A S$, $v_{x}: B_{\infty} \rightarrow B$ and $v_{x}^{0}: B_{0} \rightarrow K$ by $u_{x}(y)=x y$ for $y \in T, v_{x}(b)=b x$ for $b \in B_{\infty}$ and $v_{x}^{0}=\left.v_{x}\right|_{B_{0}}$, the restriction of $v_{x}$ to $B_{0}$, respectively. Then the following theorem gives a characterization of $B_{a}$ and $K_{a}$ in terms of these operators.

Theorem 3.3. Let $x$ be an infinite matrix and $u_{x}, v_{x}$ and $v_{x}^{0}$ the multiplication operators as above. Then we have:

(a) $x \in B_{a}$ if and only if any one of these operators is defined and bounded;

(b) $x \in K_{a}$ if and only if any one of these operators is defined and compact.

Moreover, if $x \in B_{a}$, then $\left\|u_{x}\right\|=\left\|v_{x}\right\|=\left\|v_{x}^{0}\right\|=\|x\|_{a}$.

Proof. Let $x \in B_{a}$. Then the mapping $v_{x}: b \mapsto b x$ is bounded as an operator from $B_{\infty}$ to $B$ by Proposition 2.1, and consequently $v_{x}^{0}=\left.v_{x}\right|_{B_{0}}$ is a bounded operator 
from $B_{0}$ to $B$. We now show that in fact $v_{x}^{0}\left(B_{0}\right) \subset K$. Let $b \in B_{0}$ and $\epsilon>0$. Then, for $n$ big enough, we have by Proposition 2.1

$$
\begin{aligned}
& \left\|b\left(x-\sum_{k=1}^{n} x_{(k)}\right)\right\|=\left\|\left(b-\sum_{k=1}^{n} b_{(k)}\right) x\right\| \leq\left\|\left(b-\sum_{k=1}^{n} b_{(k)}\right) x\right\|_{a} \\
& \leq\left\|b-\sum_{k=1}^{n} b_{(k)}\right\|_{\infty}\|x\|_{a} \leq \epsilon\|x\|_{a} .
\end{aligned}
$$

It follows that $\left\|b x-\sum_{k=1}^{n}(b x)_{(k)}\right\| \rightarrow 0$ as $n \rightarrow \infty$ and, hence, $b x \in K$. So, $v_{x}^{0}: B_{0} \rightarrow K$ is bounded, and then also its Banach adjoint $\left(v_{x}^{0}\right)^{*}$ is bounded as an operator from $T=K^{*}$ to $A S=B_{0}^{*}$. But now it is straightforward to prove (from the definition) that $\left(v_{x}^{0}\right)^{*}$ is again a multiplication operator (from $T$ to $A S$ ), equal to $u_{x}$. In the same way we find that $\left(u_{x}\right)^{*}=v_{x}$.

On the other hand, if any of the operators in (i), (ii) or (iii) is bounded, the same is true for the others (by duality), and all operators have the same norm. In particular, we have $b x \in B$ for every $b \in B_{\infty}$, and this is equivalent to $x \in B_{a}$ (by Theorem 2.5). Therefore, (a) is proved.

To prove (b), suppose $v_{x}$ is a compact operator. Then for every $b \in B_{\infty}$ the sequence $\left\{\sum_{k=1}^{n} b_{(k)}\right\}$ is bounded in $B_{\infty}$, and it is possible to choose a sequence $\left\{\sum_{k=1}^{n_{j}} b_{(k)} x_{(k)}\right\}$ which is convergent in $B$ (necessarily towards $b x$ because of its strong convergence to $b x)$. So, $b x=\lim _{j} \sum_{k=1}^{n_{j}}(b x)_{(k)} \in K$ for every $b \in B_{\infty}$, and hence $x \in K_{a}$. The converse is also true by Proposition 2.1(d), since in this case $v_{x}$ can be approximated by finite rank operators. Namely, $\left\|v_{x} b-\sum_{k=1}^{n} v_{x_{(k)}} b\right\| \leq$ $\|b\|_{\infty}\left\|x-\sum_{k=1}^{n} x_{(k)}\right\|_{a}$ for every $b \in B_{\infty}$, and hence $\left\|v_{x}-\sum_{k=1}^{n} v_{x_{(k)}}\right\| \rightarrow 0$ as $n \rightarrow \infty$. So, $v_{x}$ is compact if and only if $x \in K_{a}$. By using the theorem of Schauder it follows that $u_{x}$ and $v_{x}^{0}$ are also compact if and only if $x \in K_{a}$.

Since the map $b \mapsto b x$ from $B_{\infty}$ to $B$ is closed, the boundedness of $v_{x}$ follows merely from the fact that $b x \in B$ for every $b \in B_{\infty}$ (by the Closed Graph Theorem). The same is true for the map $b \mapsto b x$ from $B_{0}$ to $B$ (or to $K$ ) or for the map $y \mapsto x y$ from $T$ to $A S$. Hence, using the general notation $M(X, Y)$ for the space of all matrices multiplying pointwise $X$ into $Y$ and the notation $C M(X, Y)$ for the space of all matrices representing compact multiplication operators from $X$ to $Y$, we have the following consequence.

Corollary 3.4. Using the above notation we have:

(a) $B_{a}=M\left(B_{\infty}, B\right)=M\left(B_{0}, B\right)=M\left(B_{0}, K\right)=M(T, A S)$;

(b) $K_{a}=C M\left(B_{\infty}, B\right)=C M\left(B_{0}, B\right)=C M\left(B_{0}, K\right)=C M(T, A S)$.

Proof. We shall only show that $M\left(B_{0}, B\right)$ is equal to the rest in (a) (and similary for $C M\left(B_{0}, B\right)$ in (b)); other equalities are the ingredient of Theorem 3.3. We obviously have $M\left(B_{\infty}, B\right) \subset M\left(B_{0}, B\right)$. On the other hand, we can prove as before that $b x \in K$ for every $x \in M\left(B_{0}, B\right)$. Hence, $\left(v_{x}^{0}\right)^{*}=u_{x}$ is well defined and bounded as an operator from $T$ to $A S$, and the equality follows from Theorem 3.3 .

Remark 3.5. It is well known that an operator between two Banach spaces $X$ and $Y$ is weakly compact if and only if its second adjoint maps $X^{* *}$ into (the canonical image of) $Y$ in $Y^{* *}$. As $x \in K_{a}$ is equivalent to $b x \in K$ for every $b \in B_{\infty}$ by Theorem 2.7, this means that $v_{x}^{0}: B_{0} \rightarrow K$ is weakly compact. Thus, in light 
of Theorem 3.3(b), we see that the multiplication operator $v_{x}^{0}$ (or $v_{x}=\left(v_{x}^{0}\right)^{* *}$ ) is weakly compact if and only if it is compact, and this happens if and only if $x \in K_{a}$.

\section{OTHER NORMS AND DUALITY}

The Banach algebra $M\left(B_{a}, A S\right)$, introduced in [3] (see Definition 3.1), turns out to play a similar role in the duality of absolutely bounded and absolutely compact matrices as $T$ plays in the duality of bounded and compact matrices. A natural algebra norm on $M\left(B_{a}, A S\right)$ is given by $\|y\|_{M\left(B_{a}, A S\right)}=\sup \left\{\|x y\|_{A S} ; x \in\right.$ $\left.B_{a},\|x\|_{a} \leq 1\right\}$. It is straightforward to prove that $M\left(B_{a}, A S\right)$ is a Banach algebra in the norm $\|\cdot\|_{M\left(B_{a}, A S\right)}$, but we omit the proof since it is given in 3] for a larger class of algebras (see Theorem 3.2).

The following duality theorem is also proven in [3] in a more general situation (see [3, Theorem 3.10 and Theorem 3.14).

Theorem 4.1. We have $K_{a}^{*}=M\left(B_{a}, A S\right)$ and $M\left(B_{a}, A S\right)^{*}=B_{a}$.

Corollary 4.2. $B_{a}^{*}=M\left(B_{a}, A S\right) \oplus\left(K_{a}\right)^{\perp}$, where $\left(K_{a}\right)^{\perp}$ is the annihilator in $B_{a}^{*}$ of $K_{a} \subset B_{a}$.

Another nice algebra, also introduced in $[3$, is $M(B, A S)$. We relate both algebras to the trace class $T$.

Proposition 4.3. $M(B, A S) \subset T \subset M\left(B_{a}, A S\right)$ with both inclusions proper.

Proof. The proper inclusion $M(B, A S) \subset T$ is proven in [3] (see Corollary 2.4 and Example 2.5). The inclusion $T \subset M\left(B_{a}, A S\right)$ is clear from Theorem 3.3(a). Here the equality cannot hold, as can be seen from the counterexample $y=\bigoplus_{n=1}^{\infty} u_{n} / n^{2}$ with the usual unitary matrices $u_{n}$ defined in Remark 2.4. In this case $y \notin T$ while $|y| \in T$, so that $|y| \in M\left(B_{a}, A S\right)$ and, hence, also $y \in M\left(B_{a}, A S\right)$.

We conclude with two theorems giving a characterization of elements in $M\left(B_{a}, A S\right)$ and $M(B, A S)$ in terms of the unconditional convergence of the standard decomposition, similar to the characterization of absolutely compact matrices in Theorem 3.1.

Theorem 4.4. For a matrix $y$ with the standard decomposition $y=\sum_{k} y_{(k)}$, the following assertions are equivalent:

(i) $y \in M\left(B_{a}, A S\right)$;

(ii) $y=\sum_{k} y_{(k)}$ is unconditionally convergent in the norm $\|\cdot\|_{M\left(B_{a}, A S\right)}$;

(iii) $|y|=\sum_{k}|y|_{(k)}$ is convergent in the norm $\|\cdot\|_{M\left(B_{a}, A S\right)}$.

Proof. Note first that $b y \in M\left(B_{a}, A S\right)$ for every $b \in B_{\infty}$ and $y \in M\left(B_{a}, A S\right)$ and that $\|b y\|_{M\left(B_{a}, A S\right)} \leq\|b\|_{\infty}\|y\|_{M\left(B_{a}, A S\right)}$. From this we can immediately derive that $y \in M\left(B_{a}, A S\right)$ if and only if $|y| \in M\left(B_{a}, A S\right)$.

If $y \in M\left(B_{a}, A S\right)$, it follows from the proofs of Lemma 3.6 and Lemma 3.7 in [3] that the series $y=\sum_{k} y_{(k)}$ converges in the norm $\|\cdot\|_{M\left(B_{a}, A S\right)}$. Replacing $y$ with $b y$ where $b \in B_{\infty}$ and using the known characterization of the unconditional summability from [2] we see that the convergence of the series $y=\sum_{k} y_{(k)}$ is unconditional. The implications (ii) $\Longrightarrow$ (iii) and (iii) $\Longrightarrow$ (i) are then trivial by the remark in the first paragraph.

Note that by Theorem 4.4(ii), the ideal of all matrices with finite support is dense in $M\left(B_{a}, A S\right)$ in the norm of $M\left(B_{a}, A S\right)$ and, hence, also $M(B, A S)$ and $T$ are dense in $M\left(B_{a}, A S\right)$. 
Theorem 4.5. For a matrix $y$ with the standard decomposition $y=\sum_{k} y_{(k)}$, the following assertions are equivalent:

(i) $y \in M(B, A S)$;

(ii) $y \in M\left(B_{\infty}, T\right)$;

(iii) $y=\sum_{k} y_{(k)}$ is (weakly) unconditionally convergent in the trace class $T$.

Proof. The equivalence (i) $\Longleftrightarrow$ (ii) can be obtained along the same lines as in proving previous theorems by the use of elementary duality theory. Note that (i) is equivalent to $\sum_{i, j}\left|x_{i j} y_{i j}\right|<\infty$ for every $x \in B$, and this is essentially the weak unconditional convergence of the series in (iii). On the other hand, (ii) means the bounded multiplier convergence of the standard decomposition for $y \in T$ and is equivalent to its unconditional convergence by the general theory (see [2]).

\section{ACKNOWLEDGEMENTS}

The author thanks Professor Bojan Magajna for reading the first version of this paper and suggesting several improvements and the referee for many useful comments.

\section{REFERENCES}

[1] P. Chaisuriya, S.-C. Ong, Absolute Schur algebras and unbounded matrices, SIAM J. Matrix Anal. Appl. 20 (1999), 596-605. MR.1685044(2000g:15023)

[2] J. Diestel, H. Jarchow, A. Tonge, Absolutely summing operators, Cambridge University Press, 1995. MR1342297 (96i:46001)

[3] L. Livshits, S.-C. Ong, S.-W. Wang, Banach space duality of absolute Schur algebras, Integral Equations Operator Theory 41 (2001), 343-359. MR1853675 (2002f:46087)

[4] I. Schur, Bemerkungen zur Theorie der beschränkten Bilinearformen mit unendlich vielen Veränderlichen, J. Reine Angew. Math. 140 (1911), 1-28.

[5] V.S. Sunder, Absolutely bounded matrices, Indiana Univ. Math. J. 27 (1978), 919-927. MR511247 (80d:47052)

University of Ljubluana, IMFM, Jadranska Ul. 19, 1000 Ljubluana, Slovenia

E-mail address: milan.hladnik@fmf.uni-lj.si 